\title{
Parametrization of acoustic boundary absorption and dispersion properties in time-domain source/receiver reflection measurement
}

\author{
Adrianus T. de Hoop, ${ }^{\text {a) }}$ Chee-Heun Lam, ${ }^{\text {b) }}$ and Bert Jan Kooij \\ Laboratory of Electromagnetic Research, Faculty of Electrical Engineering, Mathematics and Computer \\ Science, Delft University of Technology, 4 Mekelweg, 2628 CD Delft, The Netherlands
}

(Received 30 September 2004; revised 8 March 2005; accepted 25 May 2005)

\begin{abstract}
Closed-form analytic time-domain expressions are obtained for the acoustic pressure associated with the reflection of a monopole point-source excited impulsive acoustic wave by a planar boundary with absorptive and dispersive properties. The acoustic properties of the boundary are modeled as a local admittance transfer function between the normal component of the particle velocity and the acoustic pressure. The transfer function is to meet the conditions for linear, time-invariant, causal, passive behavior. A parametrization of the admittance function is put forward that has the property of showing up explicitly, and in a relatively simple manner, in the expression for the reflected acoustic pressure. The partial fraction representation of the complex frequency domain admittance is shown to have such a property. The result opens the possibility of constructing inversion algorithms that enable the extraction of the relevant parameters from the measured time traces of the acoustic pressure at different offsets, parallel as well as normal to the boundary, between source and receiver. Illustrative theoretical numerical examples are presented. (C) 2005 Acoustical Society of America. [DOI: 10.1121/1.1954567]
\end{abstract}

PACS number(s): 43.20.Bi, 43.20.El, 43.20.Px, 43.55.Ev [JJM] Pages: 654-660

\section{INTRODUCTION}

In a variety of applications in acoustics (for example, in outdoor sound propagation, traffic noise analysis, jet-engine sound absorption in aircraft engineering and architectural acoustics), the analysis of the point-source excited reflection of sound waves by a boundary surface with certain absorptive and dispersive properties is of interest. In all these cases, the absorptive and dispersive properties of the boundary need characterization by a judiciously chosen set of parameters. Following the pioneering paper by Ingard (1951), such a characterization goes via a local acoustic admittance, i.e., via a linear, time-invariant, causal, passive transfer function that links the normal component of the particle velocity on the boundary to the local acoustic pressure. For the canonical configuration consisting of a planar boundary, a monopole acoustic (volume injection) source and a monopole acoustic (pressure) point receiver, we derive closed-form time-domain expressions for the received signal. For the same configuration and along similar lines, a recent paper (Lam et al., 2004) discusses some ad-hoc cases, where the boundary's properties are expressed via a complex-frequency domain Padé representation, the coefficients in which are matched to experimental data available in the literature. The approach via the Padé representation appears, however, to be limited to at most the Padé $(2,2)$ one. In the present paper, a more systematic approach is followed where the complex-frequency domain characterization of the boundary admittance goes via

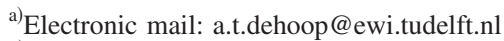

${ }^{b)}$ Presently at Laboratory of Circuits and Systems, Faculty of Electrical Engineering, Mathematics and Computer Science, Delft University of Technology, 4 Mekelweg, 2628 CD Delft, The Netherlands.
}

a partial-fraction representation that allows the incorporation of an arbitrary number of terms, each of them with an interpretable influence on the received signal. Amongst others, it is shown that, when source and receiver are both close to the boundary and the terms in the partial-fraction admittance representation meet a certain condition, large-amplitude oscillatory surface effects can occur. Their amplitudes can even exceed the acoustic pressure values associated with the reflection against a perfectly rigid boundary, a phenomenon that has also been reported elsewhere in the literature (Wenzel, 1974; Thomasson, 1976; Donato, 1976a, 1976b) and is confirmed by pertaining experiments (Daigle et al., 1996) as well as by computational finite-difference time-domain and finite element method studies (Ju and Fung; 2002; Van den Nieuwenhoff and Coyette, 2001).

The analysis is carried out with the aid of an extension (De Hoop, 2002) of the senior (first) author's modification of the Cagniard method (Cagniard, 1962; De Hoop, 1960; De Hoop and Van der Hijden, 1984). It yields closed-form analytic expressions for the time-domain acoustic pressure in the model configuration under investigation. Not only do these expressions reveal how the parameters governing the absorption and dispersion properties of the reflecting boundary show up in the measured acoustic pressure, but they can also serve as benchmarks in further computational studies based on the numerical discretization of the acoustic wave equations.

The methodology leans heavily on the use of the Schouten-Van der Pol theorem of the unilateral Laplace transformation (Schouten, 1934, 1961; Van der Pol, 1934; Van der Pol and Bremmer, 1950). This theorem interrelates two (causal) functions of time whose (unilateral) Laplace 
transforms are related such that the Laplace transform of the latter arises out of the Laplace transform of the former upon replacing the transform parameter $s$ with a certain function $\phi(s)$, where $\phi(s)$ belongs to the class of functions for which a causal time function corresponding to $\exp [-\phi(s) \tau]$, with $\tau \geqslant 0$, exists.

The analysis can be carried out for an arbitrary number of terms in the partial-fraction characterization of the boundary's acoustic admittance, each of them provided with its associated two parameters. This implies that a rather accurate tuning of the parameters to match the measured values of the admittance (a procedure that is usually carried out in the frequency domain) can be achieved by incorporating a sufficient number of terms.

Some theoretical numerical examples illustrate how some physical phenomena can be attributed to certain ranges of the values of the parameters involved.

\section{FORMULATION OF THE PROBLEM}

Position in the configuration is specified by the coordinates $\{x, y, z\}$ with respect to an orthogonal, Cartesian reference frame with the origin $\mathcal{O}$ and the three mutually perpendicular base vectors $\left\{\boldsymbol{i}_{x}, \boldsymbol{i}_{y}, \boldsymbol{i}_{z}\right\}$ of unit length each; they form, in the indicated order, a right-handed system. The position vector is $\boldsymbol{r}=x \boldsymbol{i}_{x}+y \boldsymbol{i}_{y}+z \boldsymbol{i}_{z}$. The vectorial spatial differentiation operator is $\boldsymbol{\nabla}=\boldsymbol{i}_{x} \partial_{x}+\boldsymbol{i}_{y} \partial_{y}+\boldsymbol{i}_{z} \partial_{z}$. The time coordinate is $t$; differentiation with respect to time is denoted by $\partial_{t}$.

The acoustic wave motion is studied in the half-space $\mathcal{D}=\{-\infty<x<\infty,-\infty<y<\infty, 0<z<\infty\}$, which is filled with a fluid with volume density of mass $\rho_{0}$ and compressibility $\kappa_{0}$. The speed of sound waves in it is $c_{0}=\left(\rho_{0} \kappa_{0}\right)^{-1 / 2}$. The acoustic wave motion is excited by an acoustic monopole point source with volume injection rate $Q_{0}(t)$ and located at $\boldsymbol{r}_{0}=\{0,0, h\}$, with $h \geqslant 0$. We assume that $Q_{0}(t)=0$ for $t<0$. The acoustic pressure $p(\boldsymbol{r}, t)$ and the particle velocity $\boldsymbol{v}(\boldsymbol{r}, t)$ then satisfy the first-order acoustic wave equations (De Hoop, 1995, p. 44)

$$
\begin{aligned}
& \boldsymbol{\nabla} p+\rho_{0} \partial_{t} \boldsymbol{v}=\mathbf{0}, \\
& \boldsymbol{\nabla} \cdot \boldsymbol{v}+\kappa_{0} \partial_{t} p=Q_{0}(t) \delta\left(\boldsymbol{r}-\boldsymbol{r}_{0}\right) .
\end{aligned}
$$

Causality entails that $p(\boldsymbol{r}, t)=0$ and $\boldsymbol{v}(\boldsymbol{r}, t)=\mathbf{0}$ for $t<0$ and all $\boldsymbol{r} \in \mathcal{D}$. The acoustic properties of the planar boundary are modeled via the local, linear, time-invariant, causal, passive acoustic admittance relation

$$
v_{z}(x, y, 0, t)=-\left(\rho_{0} c_{0}\right)^{-1} Y\left({ }^{(t)} * p(x, y, 0, t),\right.
$$

$$
(t)
$$

where * denotes time convolution and $Y(t)$ is the boundary's acoustic time-domain admittance transfer function, normalized with respect to the acoustic plane-wave admittance $\left(\rho_{0} c_{0}\right)^{-1}$ of the fluid. Figure 1 shows the configuration.

The acoustic wave field in the fluid is written as the superposition of the incident wave field to be denoted by the superscript $i$ and the reflected wave field to be denoted by the superscript $r$. The incident wave field is the wave field that is

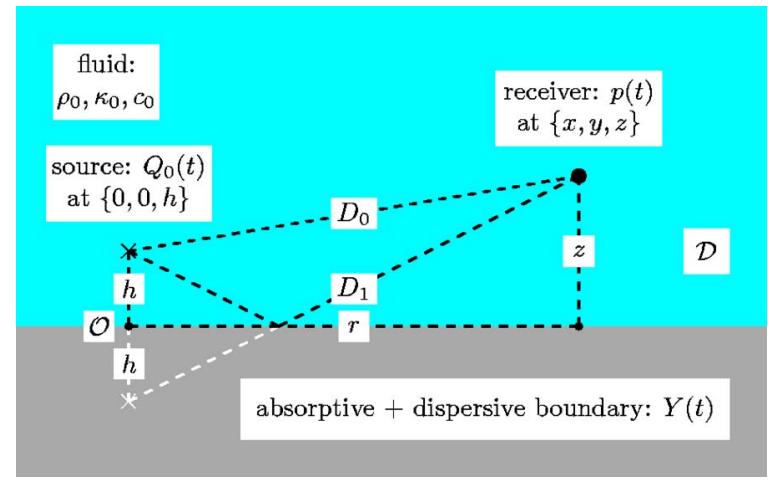

FIG. 1. Fluid-filled half-space with volume injection point source, acoustic pressure point receiver, and reflecting absorptive and dispersive boundary.

generated by the source and would be the total wave field in the absence of the boundary. Its acoustic pressure satisfies the scalar wave equation

$$
\nabla^{2} p^{i}-c_{0}^{-2} \partial_{t}^{2} p^{i}=-\rho_{0} \partial_{t} Q_{0}(t) \delta(x, y, z-h) .
$$

From this equation we obtain (see, for example, De Hoop, 1995, pp. 93-97)

$$
p^{i}(\boldsymbol{r}, t)=\rho_{0} \partial_{t}^{2} Q_{0}\left({ }^{(t)}\right)^{i} G^{i}(\boldsymbol{r}, t),
$$

in which the incident-wave Green's function is

$$
G^{i}(\boldsymbol{r}, t)=\frac{H\left(t-T_{0}\right)}{4 \pi D_{0}} \text { for } D_{0}>0,
$$

with

$$
D_{0}=\left[x^{2}+y^{2}+(z-h)^{2}\right]^{1 / 2} \geqslant 0
$$

as the distance from the source to the receiver,

$$
T_{0}=D_{0} / c_{0}
$$

as the travel time from source to receiver and $H(t)$ as the Heaviside unit step function.

\section{THE COMPLEX SLOWNESS REPRESENTATION FOR THE ACOUSTIC WAVE FIELDS}

The time invariance of the configuration and the causality of the sound waves are taken into account by the use of the unilateral Laplace transform:

$$
\{\hat{p}, \hat{\boldsymbol{v}}\}(\boldsymbol{r}, s)=\int_{t=0}^{\infty} \exp (-s t)\{p, \boldsymbol{v}\}(\boldsymbol{r}, t) d t .
$$

The Laplace transform parameter $s$ is taken positive and real. Then, according to Lerch's theorem (Widder, 1946) a oneto-one mapping exists between $\{p, \boldsymbol{v}\}(\boldsymbol{r}, t)$ and their timeLaplace transformed counterparts $\{\hat{p}, \hat{\boldsymbol{v}}\}(\boldsymbol{r}, s)$. The fluid is initially at rest, which has the consequence that the transformation property $\partial_{t} \rightarrow s$ holds. Next, the complex slowness representations for $\{\hat{p}, \hat{\boldsymbol{v}}\}(\boldsymbol{r}, s)$ are introduced as 


$$
\begin{aligned}
\{\hat{p}, \hat{\boldsymbol{v}}\}(x, y, z, s)= & \frac{s^{2}}{4 \pi^{2}} \int_{\alpha=-\infty}^{\infty} d \alpha \int_{\beta=-\infty}^{\infty}\{\tilde{p}, \widetilde{\boldsymbol{v}}\}(\alpha, \beta, z, s) \\
& \times \exp [-i s(\alpha x+\beta y)] d \beta,
\end{aligned}
$$

where $\alpha$ and $\beta$ are the wave slownesses in the $x$ and $y$ directions, respectively. This representation entails the properties $\partial_{x} \rightarrow-i s \alpha, \partial_{y} \rightarrow-i s \beta$. Use of the transforms in Eqs. (1)-(4) yields for the incident wave

$$
\widetilde{p}^{i}(\alpha, \beta, z, s)=\frac{\rho_{0} \hat{Q}_{0}(s)}{2 \gamma_{0}} \exp \left(-s \gamma_{0}|z-h|\right),
$$

while for the reflected wave we write

$$
\widetilde{p}^{r}(\alpha, \beta, z, s)=\frac{\rho_{0} \hat{Q}_{0}(s)}{2 \gamma_{0}} \widetilde{R} \exp \left[-s \gamma_{0}(z+h)\right],
$$

in which

$$
\gamma_{0}(\alpha, \beta)=\left(c_{0}^{-2}+\alpha^{2}+\beta^{2}\right)^{1 / 2} \quad \text { with } \operatorname{Re}\left(\gamma_{0}\right) \geqslant 0
$$

is the wave slowness normal to the boundary and $\widetilde{R}$ denotes the slowness-domain reflection coefficient. Use of Eqs. (11) and (12) in the complex slowness domain counterpart of the admittance boundary condition (3), together with the property [cf. Eq. (1)]

$$
\widetilde{v}_{z}=-\left(s \rho_{0}\right)^{-1} \partial_{z} \widetilde{p},
$$

Eqs. (11), (12), and (14) lead to

$$
\left(\gamma_{0} / \rho_{0}\right)(1-\widetilde{R})=\left(\rho_{0} c_{0}\right)^{-1} \hat{Y}(s)(1+\widetilde{R}),
$$

from which it follows that

$$
\widetilde{R}=\frac{c_{0} \gamma_{0}-\hat{Y}(s)}{c_{0} \gamma_{0}+\hat{Y}(s)}=1-\frac{2 \hat{Y}(s)}{c_{0} \gamma_{0}+\hat{Y}(s)} .
$$

\section{SPACE-TIME EXPRESSIONS FOR THE ACOUSTIC WAVE FIELD CONSTITUENTS}

The expressions for the time Laplace transformed reflected wave field quantities are written as

$$
\begin{aligned}
& \hat{p}^{r}(\boldsymbol{r}, s)=\rho_{0} s^{2} \hat{Q}_{0}(s) \hat{G}^{r}(\boldsymbol{r}, s), \\
& \hat{\boldsymbol{v}}^{r}(\boldsymbol{r}, s)=-s \hat{Q}_{0}(s) \boldsymbol{\nabla} \hat{G}^{r}(\boldsymbol{r}, s),
\end{aligned}
$$

in which

$$
\begin{aligned}
\hat{G}^{r}(\boldsymbol{r}, s)= & \frac{1}{4 \pi^{2}} \int_{\alpha=-\infty}^{\infty} d \alpha \int_{\beta=-\infty}^{\infty} \tilde{R} \frac{1}{2 \gamma_{0}} \exp \{-s[i(\alpha x+\beta y) \\
& \left.\left.+\gamma_{0}(z+h)\right]\right\} d \beta
\end{aligned}
$$

is the time Laplace transformed reflected-wave Green's function. The time-domain counterparts of Eqs. (17)-(19) are determined with the aid of an extension (De Hoop, 2002) of the standard modified Cagniard method (De Hoop, 1960; De Hoop and Van der Hijden, 1984). First, upon writing $x$ $=r \cos (\theta), y=r \sin (\theta)$, the transformation

$$
\alpha=i p \cos (\theta)-q \sin (\theta),
$$

$$
\beta=i p \sin (\theta)+q \cos (\theta),
$$

is carried out, which for the slowness normal to the boundary leads to $\bar{\gamma}_{0}(q, p)=\left[\Omega(q)^{2}-p^{2}\right]^{1 / 2}$, with $\Omega(q)=\left(c_{0}^{-2}+q^{2}\right)^{1 / 2}$. Next, the integrand in the integration with respect to $p$ is continued analytically into the complex $p$ plane, away from the imaginary axis and, under the application of Cauchy's theorem and Jordan's lemma, the integration along the imaginary $p$ axis is replaced by one along the hyperbolic path (modified Cagniard path) consisting of $p r+\bar{\gamma}_{0}(q, p)(z$ $+h)=\tau$, together with its complex conjugate, for $T_{1}(q)<\tau$ $<\infty$, where $T_{1}(q)=\Omega(q) D_{1}$ and $D_{1}=\left[x^{2}+y^{2}+(z+h)^{2}\right]^{1 / 2}>0$ is the distance from the image of the source to the receiver, while $\tau$ is introduced as the variable of integration. In the relevant Jacobian, the relation $\partial p / \partial \tau=i \bar{\gamma}_{0} /\left[\tau^{2}-T_{1}^{2}(q)\right]^{1 / 2}$ is used. Next, Schwarz's reflection principle of complex function theory is used to combine the integrations in the upper and lower halves of the complex $p$ plane, the orders of integration with respect to $\tau$ and $q$ are interchanged, and in the resulting integration with repect to $q$, that extends over the interval $0<q<\left(\tau^{2} / D_{1}^{2}-c_{0}^{-2}\right)^{1 / 2}$, the variable of integration $q$ is replaced with $\psi$ defined through $q=\left(\tau^{2} / D_{1}^{2}-c_{0}^{-2}\right)^{1 / 2} \sin (\psi)$, with $0 \leqslant \psi \leqslant \pi / 2$. This procedure leads to

$$
\hat{G}^{r}(r, s)=\frac{1}{4 \pi D_{1}} \int_{\tau=T_{1}}^{\infty} \exp (-s \tau) \hat{K}^{\mathrm{r}}(\boldsymbol{r}, \tau, s) d \tau,
$$

in which

$$
\hat{K}^{r}(\boldsymbol{r}, \tau, s)=\frac{2}{\pi} \int_{\psi=0}^{\pi / 2} \operatorname{Re}\left[1-\frac{2 \hat{Y}(s)}{c_{0} \bar{\gamma}_{0}+\hat{Y}(s)}\right] d \psi,
$$

with

$$
\begin{aligned}
& c_{0} \bar{\gamma}_{0}=\Gamma_{1}(\boldsymbol{r}, \tau)-i \Gamma_{2}(\boldsymbol{r}, \tau) \cos (\psi), \\
& \Gamma_{1}(\boldsymbol{r}, \tau)=c_{0} \tau(z+h) / D_{1}^{2}, \\
& \Gamma_{2}(\boldsymbol{r}, \tau)=c_{0}\left(\tau^{2}-T_{1}^{2}\right)^{1 / 2} r / D_{1}^{2},
\end{aligned}
$$

is the reflected-wave kernel function and

$$
T_{1}=T_{1}(0)=D_{1} / c_{0}
$$

is the travel time from the image of the source to the receiver. Evaluation of the integral in the right-hand side of Eq. (22) yields (see the Appendix)

$$
\hat{K}^{r}(\boldsymbol{r}, \tau, s)=1-\frac{2 \hat{Y}(s)}{\left\{\left[\Gamma_{1}(\boldsymbol{r}, \tau)+\hat{Y}(s)\right]^{2}+\Gamma_{2}^{2}(\boldsymbol{r}, \tau)\right\}^{1 / 2}} .
$$

Since the right-hand side of Eq. (27) is an analytic function of $s$ in the right half $\{\operatorname{Re}(s)>0\}$ of the complex s plane, it has a causal time-domain counterpart $K^{r}(\boldsymbol{r}, \tau, t)$ that vanishes for $t<0$. In terms of the latter, Eq. (21) leads to the time-domain expression

$$
G^{r}(\boldsymbol{r}, t)=\left[\frac{1}{4 \pi D_{1}} \int_{\tau=T_{1}}^{t} K^{r}(\boldsymbol{r}, \tau, t-\tau) d \tau\right] H\left(t-T_{1}\right) .
$$

To further separate in the second term on the right-hand side of Eq. (27) the influence of the configurational parameters of the measurement setup from the influence of the parameters

de Hoop et al:: Parametrization of an acoustic admittance-boundary 
associated with the boundary's acoustic admittance on the reflected field acoustic pressure, we make use of the Schouten-Van der Pol theorem of the unilateral Laplace transformation (Schouten, 1934, 1961; Van der Pol, 1934; Van der Pol and Bremmer, 1950) and employ the Laplacetransform integral Formula (29.3.55) from Abramowitz and Stegun (1968, p. 1024), together with some elementary rules of the Laplace transformation to obtain

$$
\begin{gathered}
\frac{\hat{Y}(s)}{\left\{\left[\Gamma_{1}(\boldsymbol{r}, \tau)+\hat{Y}(s)\right]^{2}+\Gamma_{2}^{2}(\boldsymbol{r}, \tau)\right\}^{1 / 2}} \\
\quad=1-\int_{w=0}^{\infty} K_{F}(\boldsymbol{r}, \tau, w) \hat{K}_{Y}(w, s) d w,
\end{gathered}
$$

in which

$$
\hat{K}_{Y}(w, s)=\exp [-\hat{Y}(s) w] H(w)
$$

and

$$
\begin{aligned}
K_{F}(\boldsymbol{r}, \tau, w)= & \exp \left[-\Gamma_{1}(\boldsymbol{r}, \tau) w\right]\left\{\Gamma_{1}(\boldsymbol{r}, \tau) J_{0}\left[\Gamma_{2}(\boldsymbol{r}, \tau) w\right]\right. \\
& \left.+\Gamma_{2}(\boldsymbol{r}, \tau) J_{1}\left[\Gamma_{2}(\boldsymbol{r}, \tau) w\right]\right\} H(w),
\end{aligned}
$$

where $J_{0}$ and $J_{1}$ are the Bessel functions of the first kind and orders zero and one, respectively. Use of this result in Eq. (27) yields

$$
\hat{K}^{r}(\boldsymbol{r}, \tau, s)=-1+2 \int_{w=0}^{\infty} K_{F}(\boldsymbol{r}, \tau, w) \hat{K}_{Y}(w, s) d w .
$$

In terms of the (causal) time-domain counterpart $K_{Y}(w, t)$ of $\hat{K}_{Y}(w, s)$ we end up with

$$
K^{r}(\boldsymbol{r}, \tau, t)=-\delta(t)+2\left[\int_{w=0}^{\infty} K_{F}(\boldsymbol{r}, \tau, w) K_{Y}(w, t) d w\right] H(t) .
$$

Note that in this expression the space-time configurational parameters of the fluid only occur in the kernel function $K_{F}(\boldsymbol{r}, \tau, w)$, while the parameters of $Y(t)$ only occur in the kernel function $K_{Y}(w, t)$. The space-time expressions for the reflected acoustic wave field quantities are from Eqs. (17) and (18) finally obtained as

$$
\begin{aligned}
& p^{r}(\boldsymbol{r}, t)=\rho_{0} \partial_{t}^{2} Q_{0}\left({ }^{(t)}\right)^{r}(\boldsymbol{r}, t), \\
& \boldsymbol{v}^{r}(\boldsymbol{r}, t)=-\partial_{t} Q_{0}\left({ }^{(t)} * \boldsymbol{\nabla} G^{r}(\boldsymbol{r}, t) .\right.
\end{aligned}
$$

In Sec. V, an expression for $K_{Y}(w, t)$ is obtained for the case where a partial fraction parametrization of the complex frequency domain acoustic admittance $\hat{Y}(s)$ is used to specify the boundary's acoustic dispersion and absorption properties.

\section{PARTIAL-FRACTION PARAMETRIZATION OF THE COMPLEX FREQUENCY DOMAIN ACOUSTIC ADMITTANCE AND ITS COROLLARIES}

In this section an expression for the kernel function $K_{Y}(w, t)$, introduced via Eq. (30), is constructed for the case where $\hat{Y}(s)$ is parametrized through a partial fraction representation. Let

$$
\hat{Y}(s)=\sum_{n=0}^{N} \hat{Y}^{(n)}(s),
$$

with

$$
\begin{aligned}
& \hat{Y}^{(0)}(s)=Y^{\infty}, \\
& \hat{Y}^{(n)}(s)=\frac{A_{n}}{s+\alpha_{n}} \quad \text { for } n=1, \ldots, N .
\end{aligned}
$$

Since the underlying assumption of such a representation is that $\hat{Y}(s)$ arises as the causal response from a rational time differentiation operator with real-valued coefficients and a finite number of degrees of freedom, a number of properties hold (Kwakernaak and Sivan, 1991). First, $\hat{Y}(s)$ has to be real and positive for $s$ real and positive, which entails that $Y^{\infty}$ is real and $\geqslant 0$. Furthermore, $\hat{Y}(s)$ has, in general, simple poles at $s=-\alpha_{n}(n=1, \ldots, N)$ that should be located in the left half of the complex $s$-plane. As to the terms $\hat{Y}^{(n)}(n=1, \ldots, N)$ two possibilities arise: either $\alpha_{n}(n=1, \ldots, N)$ is real and $\geqslant 0$ and the residues $A_{n}(n=1, \ldots, N)$ at the poles $s=-\alpha_{n}(n$ $=1, \ldots, N)$ are real, or pairs of $\alpha_{n}(n=1, \ldots, N)$ are complex conjugate with positive real parts and the residues $A_{n}(n$ $=1, \ldots, N)$ at such pair of poles $s=-\alpha_{n}(n=1, \ldots, N)$ are each other's complex conjugate. (The case of higher-order poles is most easily handled by a limiting confluence procedure.) Equation (36) entails a representation of $\hat{K}_{Y}(w, s)$ of the form

$$
\hat{K}_{Y}(w, s)=\prod_{n=0}^{N} \hat{K}_{Y}^{(n)}(w, s),
$$

with

$$
\begin{aligned}
& \hat{K}_{Y}^{(0)}(w, s)=\exp \left(-Y^{\infty} w\right) H(w), \\
& \hat{K}_{Y}^{(n)}(w, s)=\exp \left[-Y^{(n)}(s) w\right] H(w) \text { for } n=1, \ldots, N .
\end{aligned}
$$

The time-domain counterpart of Eq. (40) is

$$
K_{Y}^{(0)}(w, t)=\exp \left(-Y^{\infty} w\right) H(w) \delta(t) .
$$

To construct the time-domain counterpart of Eq. (41) we again use the Schouten-Van der Pol theorem and employ Formula (29.3.75) of Abramowitz and Stegun (1968, p. 1026), together with some elementary rules of the time Laplace transformation to obtain

$$
\begin{aligned}
K_{Y}^{(n)}(w, t)= & H(w) \delta(t)-\exp \left(-\alpha_{n} t\right) \\
& \times\left(A_{n} w / t\right)^{1 / 2} J_{1}\left[2\left(A_{n} w t\right)^{1 / 2}\right] H(w) H(t) \\
& \text { for } n=1, \ldots, N .
\end{aligned}
$$

In terms of Eq. (43) (that also holds for complex values of 
the parameters), the time-domain counterpart of Eq. (39) follows as

$$
K_{Y}(w, t)=K_{Y}^{(0)}(w, t)^{(t)} * K_{Y}^{(1)}(w, t)^{(t)} *^{(t)}{ }^{(N)} K_{Y}^{(N)}(w, t) .
$$

In this expression each of the factors contains only two parameters, a property that can facilitate the parameter sensitivity analysis of the reflection measurement setup.

\section{PLANE-WAVE ADMITTANCE PARAMETRIZATION OF THE COMPLEX FREQUENCY DOMAIN ACOUSTIC ADMITTANCE AND ITS COROLLARIES}

In this section an expression for the kernel function $K_{Y}(w, t)$, introduced via Eq. (30), is constructed for the case where $\hat{Y}(s)$ is parametrized through a plane-wave admittance expression, applying to a fluid with volume density of mass $\rho_{1}$, compressibility $\kappa_{1}$, normalized inertia relaxation function $\hat{\alpha}_{1}(s)$, and normalized compressibility relaxation function $\hat{\beta}_{1}(s)$. Accordingly, we write (De Hoop, 1995, p. 42)

$$
\hat{Y}_{W}(s)=Y_{1}^{\infty}[\hat{X}(s)]^{1 / 2},
$$

in which

$$
Y_{1}^{\infty}=\rho_{0} c_{0}\left(\frac{\kappa_{1}}{\rho_{1}}\right)^{1 / 2}=\frac{\rho_{0} c_{0}}{\rho_{1} c_{1}},
$$

with $c_{1}=\left(\rho_{1} \kappa_{1}\right)^{-1 / 2}$ as the corresponding wave speed, is representative for the instantaneous response and

$$
\hat{X}(s)=\frac{s+\hat{\alpha}_{1}(s)}{s+\hat{\beta}_{1}(s)}
$$

is representative for the absorptive and dispersive properties. To construct the time-domain counterpart $K_{W}(w, t)$ of the corresponding kernel function

$$
\hat{K}_{W}(w, s)=\exp \left[-\hat{Y}_{W}(s) w\right]
$$

we again use the Schouten-Van der Pol theorem and employ Formula (29.3.82) of Abramowitz and Stegun (1968, p. 1026) to obtain:

$$
\hat{K}_{W}(w, s)=\int_{u=0}^{\infty} \exp [-\hat{X}(s) u] \Upsilon(w, u) d u,
$$

where

$$
Y(w, u)=\frac{Y_{1}^{\infty} w}{\left(4 \pi u^{3}\right)^{1 / 2}} \exp \left[-\frac{\left(Y_{1}^{\infty} w\right)^{2}}{4 u}\right] H(w) H(u) .
$$

Since $\hat{\alpha}_{1}(s)$ and $\hat{\beta}_{1}(s)$ are system's response functions of the linear, time-invariant, causal, passive type, $\hat{X}(s)$ admits a partial-fraction parametrization of the type (36)-(38) and the time-domain counterpart of $\exp [-\hat{X}(s) u]$ follows from Eq. (44).

\section{SOME ILLUSTRATIVE NUMERICAL EXAMPLES}

In the following, some illustrative numerical examples are presented. The source is placed at the boundary $(h=0)$. Two receiver positions are considered, viz. one at the boundary $(r>0, z=0)$, i.e., the propagation takes place parallel to

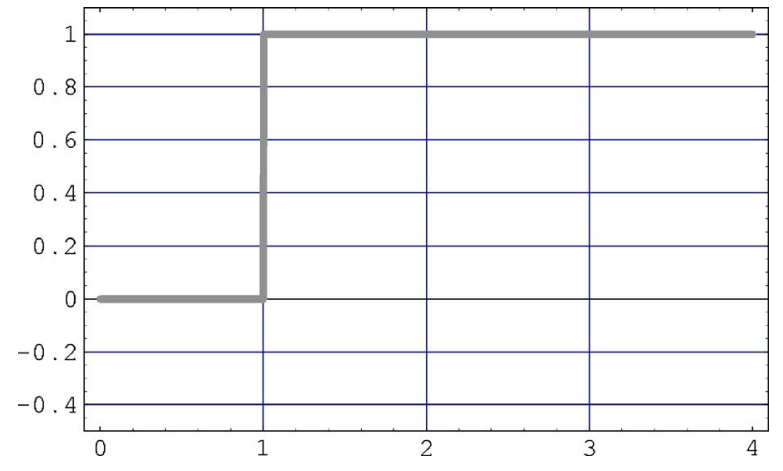

FIG. 2. Normalized incident-wave Green's function $4 \pi D_{0} G^{i}$ as a function of normalized time $t / T_{0}$.

the boundary, and one at the normal to the boundary through the source $(r=0, z>0)$, i.e., the propagation takes place normal to the boundary. With regard to the boundary's acoustic admittance, two examples are discussed: (A) the zero-order (single-term) admittance and (B) the first-order (two-terms) admittance. Figure 2 shows the normalized incident-wave Green's function as a function of normalized time [cf. Eq. (6)].

\section{A. Zero-order boundary admittance}

For the zero-order boundary admittance we have

$$
\hat{Y}(s)=Y^{\infty},
$$

which corresponds to the time-domain acoustic admittance

$$
Y(t)=Y^{\infty} \delta(t)
$$

and the time-domain boundary condition [cf. Eq.(3)]

$$
v_{z}(x, y, 0, t)=-\left(\rho_{0} c_{0}\right)^{-1} Y^{\infty} p(x, y, 0, t) .
$$

This section mainly serves to illustrate the influence of $Y^{\infty}$ on the reflection problem. Figure 3 shows the normalized reflected-wave Green's function as a function of normalized time [cf. Eqs. (27) and (28)] at (a) $r=10 \mathrm{~m}, z=0$ (propagation parallel to the boundary) and (b) $r=0, z=1 \mathrm{~m}$ (propagation normal to the boundary), for three different values of $Y^{\infty}$. Note that for propagation parallel to the boundary the normalized Green's function always starts at the value -1 , irrespective of the value of $Y^{\infty}$, while for propagation normal to the boundary the starting value is positive for $Y^{\infty}>1$, zero for $Y^{\infty}=1$ (admittance matched to the planewave value at normal incidence), and negative for $Y^{\infty}<1$.

\section{B. First-order boundary admittance}

For the first-order boundary admittance we have [cf. Eqs. (36)-(38)]

$$
\hat{Y}(s)=Y^{\infty}+\frac{A_{1}}{s+\alpha_{1}},
$$

which we rewrite as

de Hoop et al.: Parametrization of an acoustic admittance-boundary 


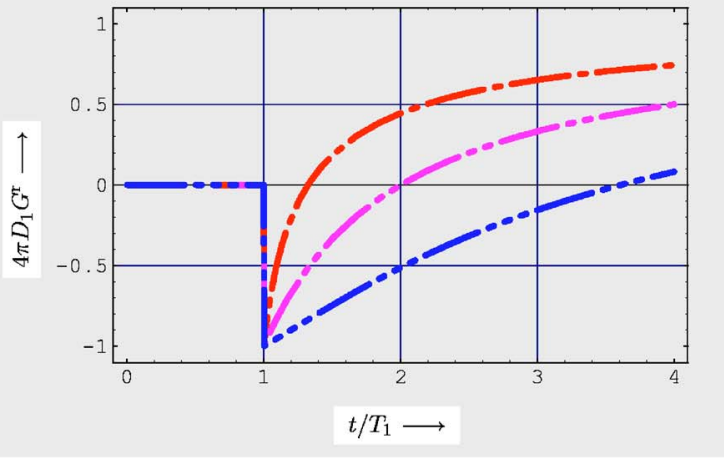

(a)

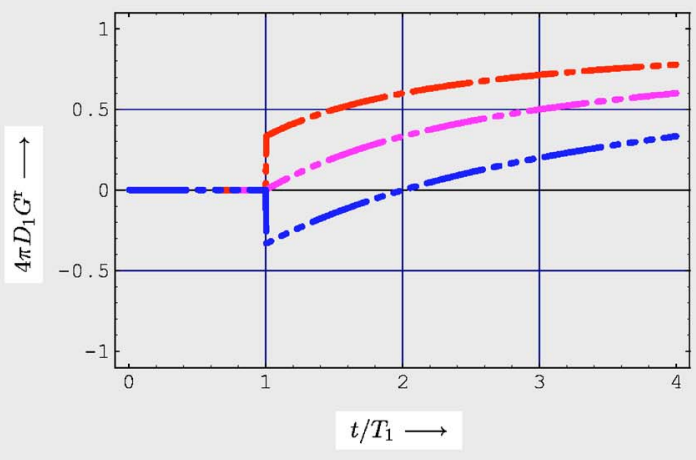

(b)

FIG. 3. Normalized reflected-wave Green's function $4 \pi D_{1} G^{r}$ as a function of normalized time $t / T_{1}$. Zero-order acoustic boundary admittance $Y=Y^{\infty}$. Source at boundary $(h=0) ; c_{0}=330 \mathrm{~m} / \mathrm{s}$. (a) Propagation parallel to boundary $(r=10 \mathrm{~m}, z=0)$, (b) propagation normal to boundary $(r=0, z=1 \mathrm{~m})$. Curves: (- . - ) $Y^{\infty}=2.0,(-. .-) Y^{\infty}=1.0$ (matched to plane-wave value at normal incidence), (- ... -) $Y^{\infty}=0.5$.

$$
\hat{Y}(s)=Y^{\infty} \frac{s+z_{1}}{s+p_{1}}
$$

where $-p_{1}=-\alpha_{1}$ is the pole of $\hat{Y}(s)$ and $-z_{1}$ is the zero of $\hat{Y}(s)$, both located in the left half of the complex $s$ plane, and

$$
A_{1}=Y^{\infty}\left(z_{1}-p_{1}\right)
$$

is the residue at the pole. Equations (54) and (55) correspond to the time-domain acoustic admittance

$$
Y(t)=Y^{\infty} \delta(t)+A_{1} \exp \left(-\alpha_{1} t\right) H(t)
$$

and the time-domain boundary condition [cf. Eq.(3)]

$$
\left(\partial_{t}+1 / \tau_{v}\right) v_{z}(x, y, 0, t)=-\left(\rho_{0} c_{0}\right)^{-1} Y^{\infty}\left(\partial_{t}+1 / \tau_{p}\right) p(x, y, 0, t),
$$

where $\tau_{v}=1 / p_{1}$ is the velocity relaxation time and $\tau_{p}=1 / z_{1}$ is the pressure relaxation time (Christensen, 2003, pp. 17-19; Meinardi, 2002, p. 105). This section mainly serves to illustrate the influence of $\tau_{v}$ and $\tau_{p}$ on the reflection problem. Therefore, we take $Y^{\infty}=1$, which implies matching to the plane-wave admittance at normal incidence.

Figure 4 shows the normalized reflected-wave Green's function as a function of normalized time [cf. Eqs. (28) and (33)] at (a) $r=10 \mathrm{~m}, z=0$ (propagation parallel to the bound-

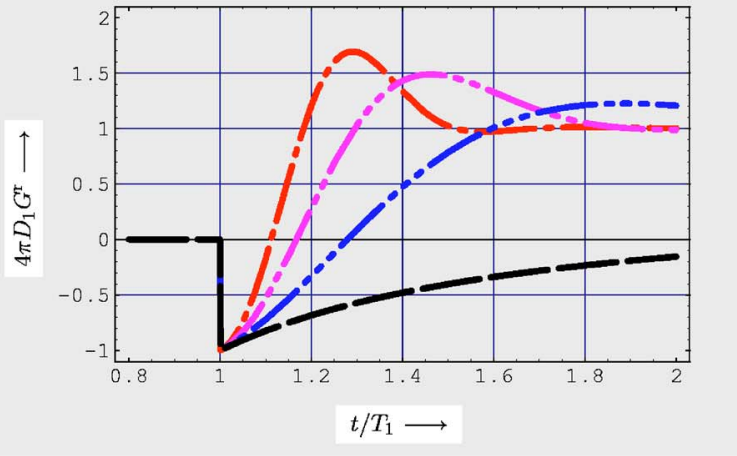

(a)

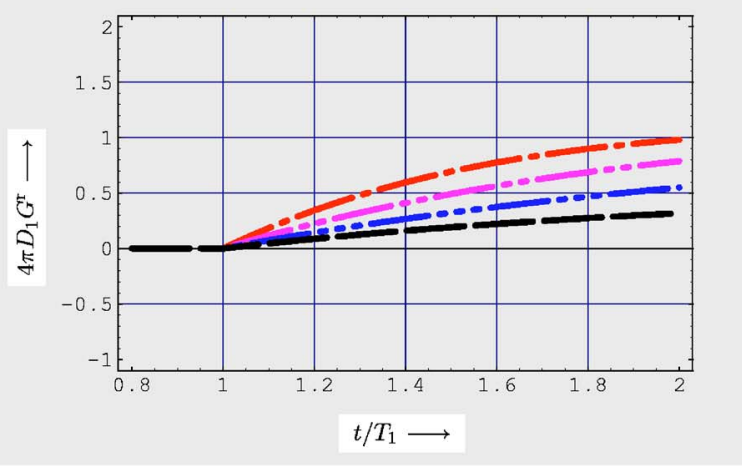

(b)

FIG. 4. Normalized reflected-wave Green's function $4 \pi D_{1} G^{r}$ as a function of normalized time $t / T_{1}$. First-order acoustic boundary admittance: $\left(\partial_{t}\right.$ $\left.+1 / \tau_{v}\right) v=-\left(\rho_{0} c_{0}\right)^{-1} Y^{\infty}\left(\partial_{t}+1 / \tau_{p}\right) p$ at boundary. Source at boundary $(h=0)$; $Y^{\infty}=1.0$ (matched to plane-wave value at normal incidence), $c_{0}=330 \mathrm{~m} / \mathrm{s}$. (a) Propagation parallel to boundary $(r=10 \mathrm{~m}, z=0)$, (b) propagation normal to boundary $(r=0, z=1 \mathrm{~m})$. Curves: $(-\ldots) \tau_{v}=1.0 \times 10^{-3} \mathrm{~s}, \tau_{p}=5.0$ $\times 10^{-2} \mathrm{~s}, A_{1}=-9.8 \times 10^{2} \mathrm{~s}^{-1} ;(-..) \tau_{v}=2.0 \times 10^{-3} \mathrm{~s}, \tau_{p}=5.0 \times 10^{-2} \mathrm{~s}, A_{1}$ $=-4.8 \times 10^{2} \mathrm{~s}^{-1} ; \quad(-\ldots-) \tau_{v}=5.0 \times 10^{-3} \mathrm{~s}, \quad \tau_{p}=5.0 \times 10^{-2} \mathrm{~s}, \quad A_{1}=-1.8$ $\left.\times 10^{2} \mathrm{~s}^{-1} ;(-)\right) \tau_{v}=1.0 \times 10^{-1} \mathrm{~s}, \tau_{p}=5.0 \times 10^{-2} \mathrm{~s}, A_{1}=1.0 \times 10^{1} \mathrm{~s}^{-1}$.

ary) and (b) $r=0, z=1 \mathrm{~m}$ (propagation normal to the boundary), for four different values of $\tau_{v}$, with $\tau_{p}$ fixed. As Fig. 4(a) shows, strong oscillations occur at propagation parallel to the boundary, which phenomenon has been referred to in Sec. I. No such oscillations show up in the propagation normal to the boundary, as Fig. 4(b) shows. It can be argued that this behavior can be inferred from Eq. (31), where $\Gamma_{1}$ is related to the offset normal to the boundary and occurs in the damping exponential function, while $\Gamma_{2}$ is related to the offset parallel to the boundary and occurs in the oscillating Bessel functions. Apparently, such an easy interpretation does not apply to Eq. (43), where for $A_{n}>0$ the Bessel functions are oscillatory, while for $A_{n}<0$ they change into modified Bessel functions of the first kind that show a monotonic behavior.

\section{DISCUSSION OF THE RESULTS}

Via the combined applications of the modified Cagniard method and the Schouten-Van der Pol theorem of the unilateral Laplace transformation the time-domain acoustic pressure of the monopole (volume injection) point-source excited wave reflected against a locally reacting, absorptive and dis- 
persive boundary has been expressed as a multiple sequence of operations acting on the source signature. Each of the kernel functions in the expression contains separately the configurational parameters of the measurement setup (location of source, receiver and boundary, and propagation through the fluid) and the parameters by which the absorptive and dispersive properties of the boundary can be characterized. Two parametrizations of the boundary's complex frequency domain acoustic admittance have been discussed in detail: the partial-fraction parametrization and the planewave admittance parametrization. The explicit attribution of a sequence of parameters to their corresponding kernel functions is conjectured to play an illuminating role in the use of the reflection measurement setup to characterize (via an appropriate inversion algorithm applied to the measured values of the acoustic pressure) the absorption and dispersion properties of the boundary, while the obtained expression itself is directly amenable to carry out the relevant parameter sensitivity analysis. It is noted that the multiple time convolutions that occur in the final expression for the acoustic pressure can numerically most profitably be evaluated through the use of the FFT algorithm.

\section{ACKNOWLEDGMENT}

The authors want to thank the (anonymous) reviewers for their constructive criticism and their suggestions for improving the paper.

\section{APPENDIX: EVALUATION OF THE INTEGRAL IN EQ. (22)}

In this Appendix the integral occurring in Eq. (22)

$$
\begin{aligned}
I & =\frac{2}{\pi} \operatorname{Re}\left[\int_{\psi=0}^{\pi / 2} \frac{1}{c_{0} \bar{\gamma}_{0}+\hat{Y}(s)} d \psi\right] \\
& =\frac{2}{\pi} \operatorname{Re}\left[\int_{\psi=0}^{\pi / 2} \frac{1}{\Gamma_{1}-i \Gamma_{2} \cos (\psi)+\hat{Y}(s)} d \psi\right] \\
& =\frac{2}{\pi} \int_{\psi=0}^{\pi / 2} \frac{\Gamma_{1}+\hat{Y}(s)}{\left[\Gamma_{1}+\hat{Y}(s)\right]^{2}+\Gamma_{2}^{2} \cos ^{2}(\psi)} d \psi,
\end{aligned}
$$

with [cf. Eqs. (24) and (25)]

$$
\begin{aligned}
& \Gamma_{1}=c_{0} \tau(z+h) / D_{1}^{2}, \\
& \Gamma_{2}=c_{0}\left(\tau^{2}-T_{1}^{2}\right)^{1 / 2} r / D_{1}^{2},
\end{aligned}
$$

is evaluated. Using the standard integral

$$
\frac{2}{\pi} \int_{\psi=0}^{\pi / 2} \frac{A}{A^{2}+B^{2} \cos ^{2}(\psi)} d \psi=\frac{1}{\left(A^{2}+B^{2}\right)^{1 / 2}},
$$

we obtain

$$
I=\frac{1}{\left\{\left[\Gamma_{1}+\hat{Y}(s)\right]^{2}+\Gamma_{2}^{2}\right\}^{1 / 2}} .
$$

This result is used in the main text.

Abramowitz, M. and Stegun, I. A. (1968). Handbook of Mathematical Functions (Dover, Mineola, NY).

Cagniard, L. (1962). Reflection and Refraction of Progressive Seismic Waves (McGraw-Hill, New York), pp. 47-50 and p. 244. [Translation and revision of Cagniard, L. (1939). Réflexion et Réfraction des Ondes Séismiques Progressives edited by E. A. Flinn and C. H. Dix (Gauthier-Villars, Paris)].

Christensen, R. M. (2003). Theory of Viscoelasticity, 2nd ed. (Dover, Mineola, NY).

Daigle, G. A., Stinson, M. R., and Havelock, D. I. (1996). "Experiments on surface waves over a model impedance plane using acoustical pulses," J. Acoust. Soc. Am. 99, 1993-2005.

De Hoop, A. T. (1960). "A modification of Cagniard's method for solving seismic pulse problems,” Appl. Sci. Res., Sect. B 8, 349-356.

De Hoop, A. T. (1995). Handbook of Radiation and Scattering of Waves (Academic, London)

De Hoop, A. T. (2002). "Reflection and transmission of a transient, elastic line-source excited SH-wave by a planar, elastic bounding surface in a solid," Int. J. Solids Struct. 39, 5379-5391.

De Hoop, A. T. and Van der Hijden, J. H. M. T. (1984). "Generation of acoustic waves by an impulsive point source in a fluid/solid configuration with a plane boundary," J. Acoust. Soc. Am. 75, 1709-1715.

Donato, R. J. (1976a). "Propagation of a spherical wave near a plane boundary with a complex impedance," J. Acoust. Soc. Am. 60, 34-39.

Donato, R. J. (1976b). "Spherical-wave reflection from a boundary of reactive impedance using a modification of Cagniard's method," J. Acoust. Soc. Am. 60, 999-1002.

Ingard, K. U. (1951). "On the reflection of a spherical sound wave from an infinite plane,” J. Acoust. Soc. Am. 23, 329-335.

Ju, H. B. and Fung, K. Y. (2002). "Time-domain simulation of acoustic sources over an impedance plane," J. Comput. Acoust. 10, 311-329.

Kwakernaak, H. and Sivan, R. (1991). Modern Signals and Systems (Prentice-Hall, Englewood Cliffs, NJ), pp. 463-466.

Lam, C. H., Kooij, B. J., and De Hoop, A. T. (2004). "Impulsive sound reflection from an absorptive and dispersive planar boundary," J. Acoust. Soc. Am. 118, 677-685

Meinardi, F. (2002). "Linear viscoelasticity", in Acoustic Interactions with Submerged Elastic Structures, edited by A. Uran, A. Boström, O. Leroy, and G. Maze, (World Scientific, Englewood Cliffs, NJ), pp. 97-126.

Schouten, J. P. (1934). "A new theorem in operational calculus together with an application of it," Physica (Amsterdam) 1, 75-80.

Schouten, J. P. (1961). Operatorenrechnung (Springer, Berlin), pp. 124-126. Thomasson, S. I. (1976). "Reflection of waves from a point source by an impedance boundary," J. Acoust. Soc. Am. 59, 780-785.

Van den Nieuwenhof, B. and Coyette, J. P. (2001). "Treatment of frequencydependent admittance boundary conditions in transient acoustic finite/ infinite-element models," J. Acoust. Soc. Am. 110, 1743-1751.

Van der Pol, B. (1934). "A theorem on electrical networks with an application to filters," Physica (Amsterdam) 1, 521-530.

Van der Pol, B. and Bremmer, H. (1950). Operational Calculus Based on the Two-sided Laplace Transform (Cambridge University Press, Cambridge, UK), pp. 232-236.

Wenzel, S. R. (1974). "Propagation of waves along an impedance boundary," J. Acoust. Soc. Am. 55, 956-963.

Widder, D. V. (1946). The Laplace Transform (Princeton University Press, Princeton, NJ), pp. 63-65. 